\title{
Acción del acetato de 6 alfa-metil 17 alfa hidroxy progesterona sobre las contracciones de Braxton-Hicks en el embarazo avanzado y algunos estudios sobre su metabolismo
}

\author{
Fernando Del Corral, M.D.++, Robert E. Stempfel, M.D., \\ and E. C. Hamhlen, M.D., Durhan, N.C.
}

(De la División de Endocrinología, Departamento de Obstetricia y Ginecología

y Departamento de Pediatría del Hospital de la Universidad de Duke).

La mayor parte de los estudios clínicos practicados con la Progesterona y sus esteroides derivados e híbridos, han estado enfocados la mayor parte de las veces sobre la acción en el endometrio y sus diversas causas de hemorragia.

A pesar de que se han hecho estudios muy variados acerca de los efectos de la progesterona en el miometrio, sinembargo se ha puesto poca atención a las respuestas que en el miometrio producen los nuevos derivados de la progesterona, activos por vía oral.

Este estudio contempla los resultados obtenidos sobre las contracciones Braxton-Hicks en el embarazo avanzado en $24 \mathrm{mu}-$ jeres normales, a las cuales se les administró oralmente acetato de 6 alfa-Metil 17 alfa-hidroxy Progesterona (Provera).

* El Dr. Harold L. Upjohn, de los Laboratorios Upjohn, Kalamazoo, Michigan, suministró atentamente la droga. (Provera).

+ Parte de los gastos de este estudio estuvieron a cargo del Consejo de Investigación de la Universidad de Duke.

++ W. K. Kellogg Fellow in Obstetrical and Gynecological Endocrinology, Duke University Medical Center (1958-1959). 
Los registros de 17 pacientes fueron escogidos para su análisis. Se hicieron además algunas determinaciones de los esteroides urinarios en un esfuerzo por investigar cambios notorios que pudieran reflejar el metabolismo y la excreción del esteroide administrado.

Se escogieron para este estudio las contracciones de Braxton Hicks en el embarazo avanzado, debido a que clínicamente es a veces imposible diferenciar una amenaza de aborto del aborto inevitable y el trabajo prematuro, del falso trabajo. No pudimos estudiar el efecto de este esteroide en las contracciones del parto, debido a que solamente disponemos de éste para uso oral, y su acción retardada por esta vía lo inhabilita para tal uso. La contractilidad del útero fué medida mediante registros hechos con el Tocógrafo de Loránd.

\section{INTRODUCCION}

Desde que Corner y asociados, ${ }^{6}$ demostraron que la actividad uterina estaba bajo el control de los ovarios, muchos investigadores han llevado a cabo una serie de experimentos con los extractos ováricos, a fin de comprobar sus efectos sobre la contracción uterina. En el mismo año Keye, ${ }^{18}$ encontró que durante el oestrus (dominación estrogénica del ciclo), las contracciones del útero de la hembra del cerdo in vitro, son amplias e infrecuentes, mientras que durante la fase luteínica (dominio progestacional), las contracciones son pequeñas y muy frecuentes. De acuerdo con Csapo, ${ }^{8}$ los hallazgos reportados por Keye están aún vigentes después de 20 años, a pesar del mejoramiento en los métodos y depuración en las técnicas empleadas. En el año de 1929, Allen y Corner, ${ }^{1}$ reportaron su observación clásica según la cual la implantación y el mantenimiento del embarazo en conejas ovariectomizadas 18 horas después de la impregnación, continuaban normalmente siempre y cuando los animales fueran inyectados con extractos de cuerpo lúteo.

Pocos años más tarde Knaus, ${ }^{19}$ usando la primera progesterona cruda, producida en Furopa, encontró que el útero bajo la influencia de los extractos del cuerpo lúteo, no respondía a la oxitocina. En la misma época Reynolds y Friedman, ${ }^{29}$ colocando un balón de caucho dentro de la cavidad uterina, pudieron demostrar que el músculo uterino está en reposo bajo la influencia del cuerpo lúteo y muy activo cuando está bajo el dominio de los 
estrógenos. Makepeace y colaboradores, ${ }^{20}$ en 1936 usando por primera vez la hormona cristalina pura, obtuvieron la misma inhibición lograda con el extracto crudo. La hormona fué llamada Progestin debido a la producción de cambios progestacionales sobre el útero de la coneja virgen castrada.

La experimentación animal, había demostrado que las contracciones uterinas inducidas por la oxitocina eran inhibidas por la progesteruna. En las hembras del gato, del perro y del cerdo de guinea, el cuerpo lúteo no es indispensable durante la segunda mitad de la gestación, mientras que en las hembras, del conejo y la rata, parece que es indispensable durante toda la época de la gestación. En la mujer y en el mono, el cuerpo lúteo no es esencial en la segunda mitad de la gestación, debido a la producción de progesterona por la placenta.

Falls y colaboradores, ${ }^{12}$ en 1936 comunicaron la supresión de las contracciones en la mujer haciendo uso de este esteroide.

Manzi, ${ }^{21}$ demostró que la progestina mantiene el útero en reposo durante el embarazo, y que éste se torna sensible a las diferentes preparaciones del lóbulo posterior de la pituitaria cuando comienza a declinar la influencia del cuerpo lúteo. Los estrógenos por otra parte, a excepción hecha, según parece del dietiletil-bestrol, sensibilizan el útero a la influencia de la oxitocina y son por lo tanto, estimulantes de la contracción uterina. ${ }^{5}$ Parkes, ${ }^{27}$ Zondek y Ascheim, ${ }^{37}$ pudieron determinar el aborto en animales administrando dosis altas de estrógenos.

Para mejor comprensión de la función accional de los esteroides ováricos sobre la contractilidad uterina, creemos aconsejable revisar brevemente el trabajo realizado por Szent-Gyorgy, ${ }^{33}$ sobre la contracción muscular. El demostró en 1945, que para obtener la contracción del músculo, se hace necesaria la unión de las proteínas Actyn y Myosin con el fin de formar así el complejo fibro-proteínico Actomyosin.

Para que esta reacción tenga lugar, se hace indispensable la presencia de la energía del nucleótido trifosfato de adenosina (A. T.P.), así como también las diferentes contracciones de iones existentes en el tejido muscular. Fig. 1.

De acuerdo con Straub, ${ }^{32}$ la actividad "máxima" del miometrio, se obtiene cuando la Actomiosina contiene dos partes de Ac- 


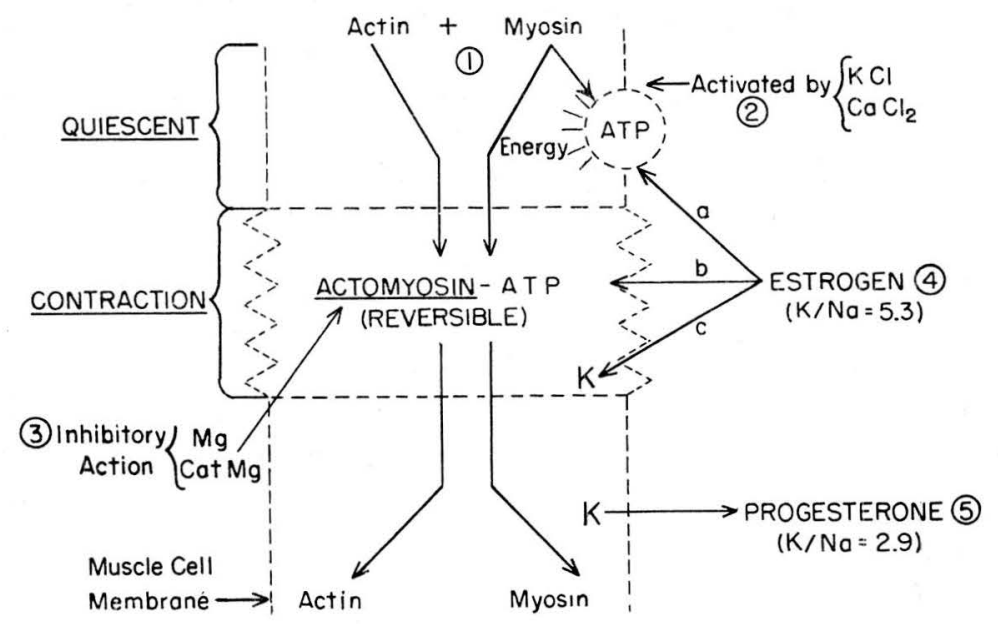

FIGURA 1 - Diagrama de los factores más importantes relacionados con la contracción de una célula muscular. Notas explicativas: 1) La unión de actin más myosin produce actomyosin en la p:esencia de energía suministrada por el ATP (trifosfato de adenosina) formando la substancia final contráctil actomyosin-ATP; 2) la acción que el myosin ejerce sobre el ATP es a tivada por $\mathrm{KC} 1$ y $\mathrm{CaC12}$; 3) Mg. solo o en combinación con calcio inhibe la acción del actomyosin; 4) los estrógenos a) reducen el "tiempo de rompimiento" del ATP, b) aumenta las concentraciones de AM (actomyosin) y ATP, c) la dominación estrogénica se tradice en concentraciones altas de $\mathrm{K}$ y bajas de $\mathrm{Na}$; 5) la progesterona "bloquea" el mecanismo de excitación y probablemente el mecanismo de excitación-contracción; el dominio progestacional se traduce en bajas concentraciones de $\mathrm{K}$ y altas concentraciones de Na.

tina por 5 de Miosina. La acción más importante de la Actomiosina (AM) es la precipitación de la contracción y la deshidratación que ocurre bajo la influencia de las sales y A.T.P. La substancia final contráctil Actomiosina A.T.P., se forma de la unión de Actomiosina A.T.P. y Actina. La Acción de la Miosina, de acuerdo con Engelhardt y asociados, ${ }^{11}$ despoja un fosfato de la molécula de A.T.P., por lo tanto tiene la propiedad de actuar como una enzima.

En otras palabras, actúa como una adenosina trifisfatasa que puede liberar la rica fuente de energía que se encuentra unida al fosfato terminal de A.T.P. energía que se libera al ser desintegrada la molécula.

La acción que la Miosina ejerce sobre el A.T.P., depende de la presencia de iones, siencio su efecto poderosamente activado por el cloruro de potasio y el cloruro de calcio. El magnesio, por el contrario, tiene una acción inhibitoria por cuanto produce una 
disociación del Actomyosin ante la presencia de trifosfato de adenosina. La combinación de magnesio y calcio tiene una acción inhibitoria sobre la contracción. ${ }^{33}$ Por otra parte, la acción del complejo final contráctil Actomyosin-A.T.P., puede ser reversible por razón del aumento o disminución en la concentración de las sales que se encuentran presentes en la fibra muscular.

De acuerdo con Csapo. ${ }^{9}$ y otros, los esteroides ováricos alteran los gradientes iónicos del miometrio. La contracción del músculo uterino depende de las concentraciones de potasio y sodio intra y extra celular, o mejor dicho, proceden de la relación en que se encuentran el sodio y el potasio tanto dentro como fuera de la céluia. Las hormonas ováricas por lo tanto actúan sobre el miometrio alterando la relación $\mathrm{K}: \mathrm{Na}$ (Fig. 1).

De acuerdo con Zarrow, ${ }^{36}$ "cuando el miometrio se encuentra dominado por la progesterona, el músculo no pierde la potencialidad de contraerse al máximo, es decir, que el mecanismo mediante el cual se produce la contracción, progresa hasta un punto muy cercano a su fin, momento en el cual el proceso es bloqueado, no obteniéndose, por lo tanto, la respuesta final contráctil". Parece desprenderse de ello, que el efecto de la progesterona no se ejerce sobre el sistema final contráctil, sino un poco antes sobre los mecanismos de excitación de la contracción. Según el mismo autor, cuando existe un dominio estrogénico en la fibra, se generan altas concentraciones de potasio dentro de la célula y bajas concentraciones de sodio dentro de la misma. Por otro lado, cuando la hormona dominante es la progesterona, la relación $\mathrm{K}: \mathrm{Na}$, se invierte, habiendo una concentración baja de potasio y alta de sodio dentro de la célula.

Horvath, ${ }^{17}$ encontró una relación potasio sodio de 5:3, bajo el dominio estrogénico y una relación $\mathrm{K}: \mathrm{Na}$ de $2: 9$ bajo el dominio de la progesterona. Fig. 1. Cuando la progesterona es la dominante, parece que todos los elementos causantes de la contracción se hallan presentes; pero ocurre que en los estados finales del proceso, se produce un bloqueo de todo el mecanismo. El útero, en consecuencia, se torra insensible a los diferentes estímulos fisiológicos y aún a la oxitocina debido a que la unión final de Actomiosina A.T.P., se bloquea.

Cuando, por alguna circunstancia, las concentraciones óptimas de la progesterona declinan, o cuando sin disminución apa- 
rente de la misma, hay un aumento de los estrógenos, el mecanismo de la contracción queda automáticamente restaurado y el útero puede desarrollar una contracción completa.

Los estrógenos por lo demás parece que actúan sobre el útero estimulando la contracción de 3 maneras diferentes: 1) Los estrógenos aumentan las concentraciones de Actomiosina (AM) y trifosfato de adenosina en la fibra muscular; (esto fue demostrado por Csapo 7, quien observó que administrando estrógenos a la coneja castrada, se producía un aumento del $120 \%$ en las concentracoines de Actomiosina, así como también una hipertrofia del músculo uterino). 2) El tiempo de ruptura o descomposición de la molécula de A.T.P. se acorta durante la terapia de estrógenos y además, la recombinación de la Actomiosina disociada, se hace más rápidamente y 3) otra acción muy importante de los estrógenos, es la inhibición que ellos ejercen sobre la progesterona cuando la relación estradiol-progesterona es mayor de $1: 200$. Se puede decir que el efecto de los estrógenos sobre la contractilidad uterina es estimulante, y no inhibitoria como algunos autores lo han afirmado. Nuestra experiencia clínica nos ha demostrado este efecto estimulante de los estrógenos en los casos en que se han empleado para sensibilizar la fibra uterina, cuando se ha hecho necesaria una inducción electiva con oxitocina. (Oportunamente en publicación posterior daremos a conocer estos resultados). Parece que existen otras substancias, como el estrofanto, que tienen también un efecto estimulante sobre la contracción uterina. ${ }^{30}$

Los efectos de la Progesterona sobre la contracción uterina podrían ser resumidos en la siguiente forma: bajo el dominio de la progesterona el miometrio es potencialmente capaz de desarrollar una tensión muscular máxima que no puede hacerse efectiva debido a que los mecanismos fisiológicos de excitación y, probablemente, el de excitación-contracción están bloqueados", por lo tanto, la actividad permanece localizada no pudiendo de este modo producirse una contracción completa. ${ }^{36}$ La progesterona disminuye la excitabilidad en una forma drástica impidiendo la propagación de la actividad a través de la membrana celular. A pesar de que el miometrio se encuentra en su máxima capacidad de trabajo, los estímulos fisiológicos que a él llegan producen sólo una respuesta localizada. ${ }^{2}$

Nosotrns no sabemos si la progesterona actúa sobre el músculo uterino directamente o por medio de sus dos isómeros, Delta 
4-3 Ketopregnane-20 alfa-ol y delta 4-3 Ketopregnane 20 beta-ol, los cuales han sido aislados recientemente. ${ }^{35}$ Los dos isómeros se han encontrado en folículos maduros, en el cuerpo lúteo, así como también en la placenta y en el tejido graso; pero ninguno de ellos se ha podido hallar en el músculo uterino, a pesar de que compuestos muy similares han sido detectados en la sangre placentaria. Ambos compuestos tienen una reconocida acción progestacional, la cual se ha hecho evidente mediante las pruebas de Hooker-Forbes y Clauberg; por lo tanto han sido considerados como gestógenos. ${ }^{35}$

Algunos autores como Csapo, citado por Bergson² y Zarrow, ${ }^{36}$ afirman que la progesterona en el segundo y tercer mes del embarazo ejeree su acción directamente desde la placenta en forma localizada. De allí es difundida luego al resto del órgano. En los animales, particularmente en el conejo, el embarazo termina cuando la acción de la progesterona llega a su fin.

En los últimos años han sido preparados comercialmente varios gestógenos o progestines, siendo alguno de ellos igual o mucho más efectivo que la progesterona. Entre estos debemos mencionar la 11-dehidroprogesterona, ${ }^{23}$ la 19 Nor-Progesterona, ${ }^{22}$ y la 17 alfa-metil progesterona..$^{15}$

\section{MATERIALES Y METODOS}

Selección de pacientes: Las pacientes embarazadas que se escogieron para esta prueba, todas pertenecían a la Clínica Prenatal de Duke University Medical Center. Veinticuatro de ellas fueron escogidas, primero, por presentar un control prenatal normal; segundo, debido a que su peso no excedía de 130 libras, factor este último muy importante para obtener un registro adecuado; tercero, por tener todas ellas un feto en presentación cefálica durante el tiempo en el cual los registros fueron hechos; y finalmente, por estar todás en las 36 o más semanas de embarazo. El tocógrafo registró, no solamente las contracciones uterinas sino también estornudes, risa, movimientos fetales y cambios en la posición de la enferma. Uno de nosotros (F. del C.) estuvo siempre presente durante todo el tiempo del registro, para evitar cualquier movimiento que pudiera interferir con éste, manteniendo a la enferma en condiciones óptimas de reposo.

Instrumento de registro: En este estudio se usó el nuevo modelo del Tocógrafo de Loránd. Este registra los cambios que ocu- 
rren durante la contracción, los cuales son transmitidos a través de la pared abdominal. El registro se hace por medio de una aguja inscriptora sobre un papel milimetrado, colocado alrededor de un cilindro giratorio. La cinta de papel con el registro gráfico de la contracción, circula a razón de $5 \mathrm{~mm}$. en 60 segundos, mediante un sistema de reloj.

Sistema de registro: La paciente, de acuerdo con lo recomendado por Murphy, ${ }^{24}$ es colocada en una posición semi-sentada, mediante una almohada colocada debajo de los hombros. Después de un reposo de 20 minutos, el tocógrafo es colocado sobre la línea media del abdomen a 5 cms. por encima del ombligo.

La duración de los registros en todas las pacientes estudiadas, fue de 1 hora, cada paciente tuvo dos registros y algunas tuvieron 3. Uno de estos sirvió de control y fue tomado antes de la administración de la droga. Cada paciente recibió una sola dosis de 6 alfa-metil 17 alfahidroxy progesterona acetato, por vía oral dosis de $20,40,50,60,70,80,90,100,150$ y $200 \mathrm{mgs}$. fueron administradas. El tiempo transcurrido entre la administración del esteroide y del segundo registro, fue escogido arbitrariamente variando desde las 16 a las 36 o más horas, siendo diferente de acuerdo con la paciente. Schofield, citado por Zarrow, ${ }^{36}$ encontró que en los animales no grávidos se requiere un tiempo promedio de 18 horas para que un progestógeno desarrolle su efecto.

\section{CARACTERISTICAS GENERALES REGISTRADAS CON EL TOCOGRAFO}

El instrumento registró contracciones uterinas de pequeña amplitud, así como también las contracciones de Braxton-Hicks, observadas ya por Murphy, ${ }^{24}$ en 1940.

Las contracciones indoloras del útero, que se presentan en las últimas semanas del embarazo, a menudo alcanzan a tener la misma magnitud de las contracciones observadas durante la primera etapa del trabajo de parto. Con el objeto de evitar el período de actividad no rítmica del útero, es aconsejable hacer un registro de la contracción después de la trigésima segunda semana de embarazo. ${ }^{24}$

Después de esta época es posible con el tocógrafo registrar las siguientes características: (Fig. 2). 


\section{CHARACTERISTIC DATA RECORDED WITH THE LORAND'S TOCOGRAPH}

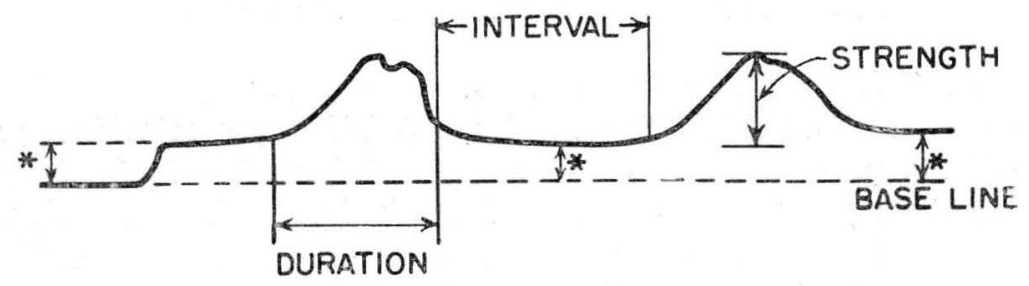

\section{TONE OF THE PARIETES}

FIGURA 2 - Características generales de la contracción registradas con el tocígrafo de Lorand.

A. - Intensidad: Esta es medida por la altura de la onda en $\mathrm{mm}$.

B. - Duración: La onda progresa gradualmente hasta su ápice donde persiste por un corto período de tiempo para luego descender gradualmente a la línea basal. La "duración" es el período de tiempo transcurrido desde el comienzo de la onda, hasta que ésta llega de nuevo a la línea basal.

C. - Intervalo: Este se mide en segundos y comprende el tiempo que transcurre desde que una onda termina, hasta el comienzo de la siguiente:

D. - Tono Parietal: Este puede ser medido en la siguiente forma: antes de que el tocógrafo sea colocado sobre el abdomen de la paciente, se permite que la aguja inscriptora trace una línea sobre la cinta, a este se le conoce con el nombre línea basal; el tocógrafo es luego colocado en su posición de registro, si el útero está suficientemente tenso, al colocarlo se produce un desplazamiento del botón abdominal, el cual levanta la aguja inscriptora de la línea basal. Para medir este tono parietal, nosotros tomamos la distancia en $\mathrm{mm}$. que existe desde los valles de la gráfica, hasta la línea basal. La distancia se toma al comienzo en la mitad $\mathrm{y}$ al final del registro obteniéndose un término medio de los tres valores, como se aprecia en la Fig. 2.

La accion de la progesterona puede ser valorada si se producen modificaciones en el: 1) tono del útero, 2) frecuencia, 3) 
intensidad, 4) duración y 5) ritmo de las diferentes contracciones uterinas. ${ }^{25}$

\section{EST UDIO DE LOS ESTEROIDES URINARIOS}

En 7 de las pacientes estudiadas se practicaron determinaciones urinarias de 17 kestosteroides, 17 hidroxy-corticoides pregnandiol y pregnanetiol antes de la administración de la droga y durante un período de 48 horas después de la administración del esteroide. Los 17 ketosteroides se dosificaron por el método de Holtorff y Koch, ${ }^{16}$ y los 17 hidroxy corticoides por el método de Glenn y Nelson. ${ }^{13}$

Uno de nosotros (R. S. S.) ${ }^{31}$, modificando la técnica de Bongiovani y Eberlein, ${ }^{3}$ determinó el pregnandiol y pregnanetriol urinarios usando columna cromatográfica con Florisil.

\section{RESULTADOS}

Se estudiaron 24 pacientes, de las cuales se obtuvieron un total de 56 gráficas. A 4 enfermas se les practicó el registro dejando transcurrir únicamente 20 horas después de la administración de la droga. Tres pacientes tuvieron su segundo registro a las 36 o más horas. Comparando los resultados de estas gráficas con los obtenidos anteriormente, observamos que la droga no es plenamente efectiva antes de las 20 horas de su ingestión y que su efecto no se mantiene después de las 36 horas.

En este estudio estamos interesados únicamente en los resultados obtenidos en el período comprendido entre las 20 y las 30 horas después de la administración oral de la hormona, por lo tanto solamente consideraremos en este informe preliminar, a 17 pacientes. Tabla $\mathrm{N}^{0} 1$.

Fue imposible determinar el tiempo óptimo en el cual la droga desarrolla su máxima acción. En relación a la dosis usada, podemos decir, que menos de $20 \mathrm{mlg}$. no tiene un efecto demostrable sobre las contracciones del embarazo avanzado; las dos pacientes que recibieron una sola dosis de $20 \mathrm{mlg}$., no presentaron modificación alguna en las características registradas, a excepción hecha de un pequeño aumento en el tono parietal. Por otra parte, parece que el aumento gradual de la dosis no produjo alteraciones de consideración, debido a que obtuvimos respuestas si- 
milares con dosis de 50 y 150 mlgs. No obstante, cinco pacientes presentaron diferentes grados de somnolencia a la mañana siguiente de administrada la droga. Todas ellas habían recibido una dosis superior a $50 \mathrm{mlg}$.

\section{EFECTOS DE LA DROGA SOBRE LAS DIFERENTES CARACTERISTICAS RECISTRADAS CON EL TOCOGRAFO}

Frecuencia: la frecuencia de las contracciones disminuyó después de administrada la droga en 9 de las 17 pacientes. En algunos casos esta disminución fue poco aparente, pero por otro lado, hubo casos en los cuales la droga suprimió hasta 13 contracciones en 1 hora. De las 17 pacientes mencionadas, únicamente 6 no presentaron modificación alguna en la frecuencia; 2 registraron un aumento en el número de contracciones; siendo en una de ellas de más de 6 contracciones por hora.

Tono Parietal: esta característica se encontró notoriamente aumentada cn la mayoría de los casos estudiados (12 de 17 pacientes). El aumento aparentemente produjo en varios casos una sensación temporal de incomodidad y de tensión abdominal.

Una de las pacientes, quien recibió una dosis de $100 \mathrm{mlg}$., anotó que sentía como una apretada faja alrededor del abdomen. A pesar de que la consistencia del útero aumentó en unos casos, el tono parietal no alcanzó a cifras mayores de $10 \mathrm{~mm}$. sobre el tono basal, a excepción hecha de 1 caso (Fig. $N^{0} 3$ ).
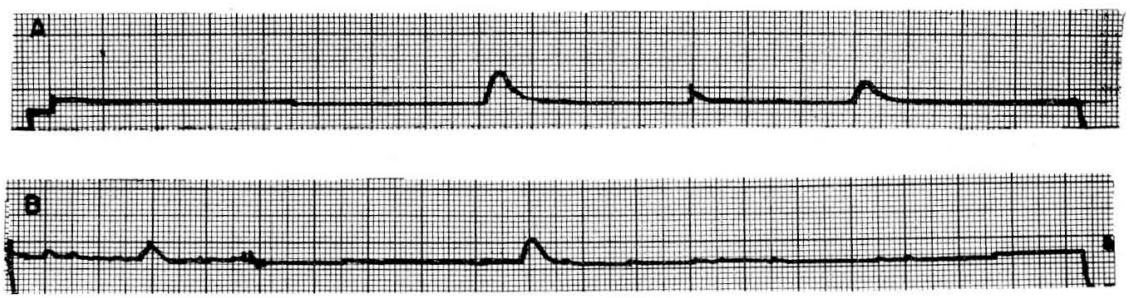

FIGURA 3 - Gráfica del paciente $\mathrm{N}^{0}$ 9. El registro A fue hecho 14 días antes del trabajo. El registro $\mathrm{B}$ fue hecho 13 días antes del trabajo y 20 horas después de la administración de $60 \mathrm{mg}$. de Provera. Nota: se observa un aumento en el tono de los parietes en el trazado B después del tratamiento. Este aumento ocurrió a pesar de que el tono original era más alto que lo corriente.

En otros el aumento del tono fué moderado como podemos ver en la figura $\mathrm{N}^{0} 4$ en el cual dicho aumento fue de $2.6 \mathrm{~mm}$. después del primer registro. En un solo caso no se presentó nin- 


\section{TABLA I}

Acción del Acetato de 6 alfa-metil 17 alfa hidroxy progesterona en las contracciones del embarazo avanzado (20 a 30 horas después de la administración del esteroide)

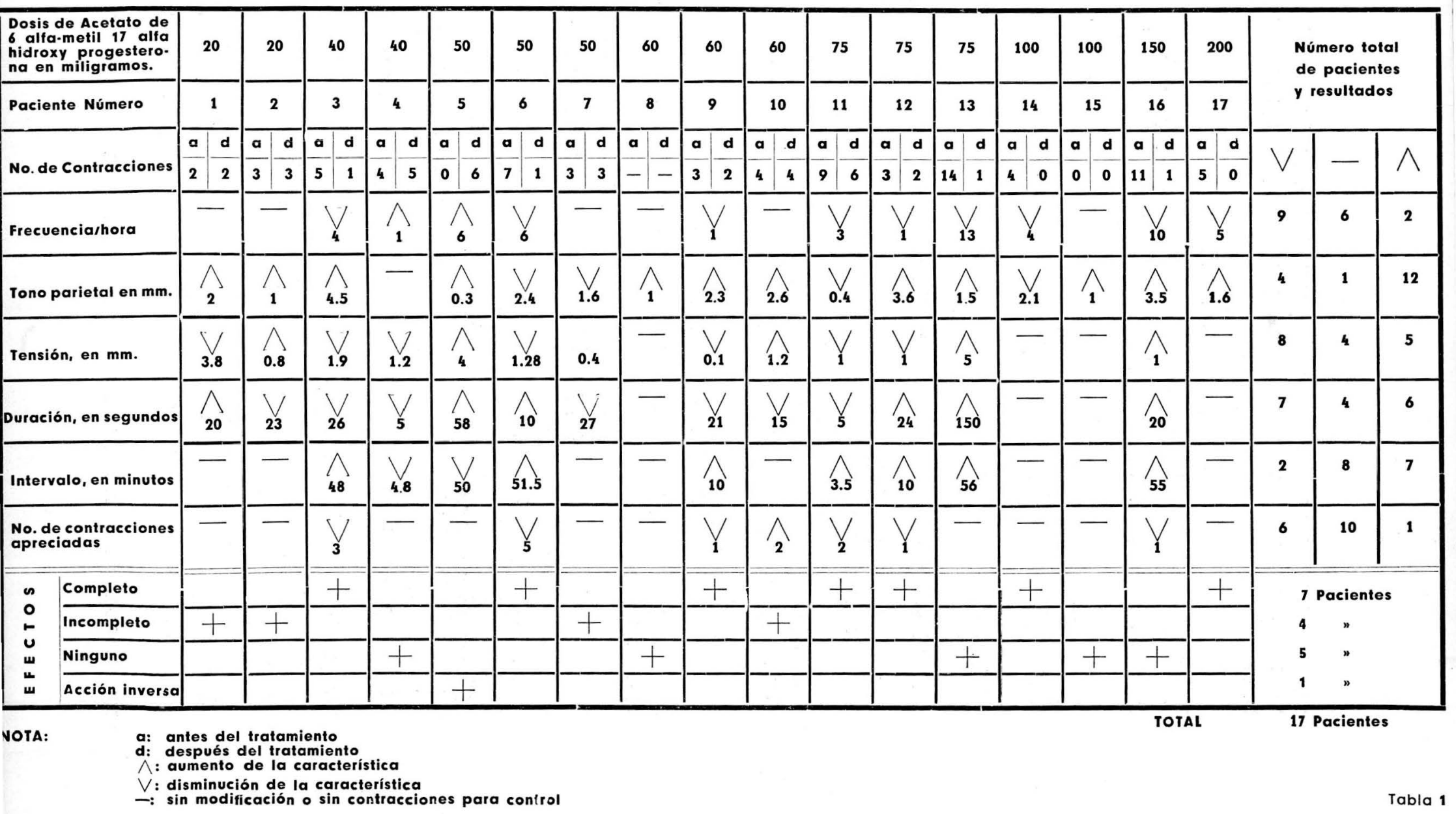


guna modificación y en los 4 restantes, se halló una pequeña disminución en el tono parietal después de administrada la hormona.

Intensidad: disminuyó en ocho casos, no se modificó en cuatro y aumentó en los cinco restantes. La disminución no fue muy aparente, pero en cambio los aumentos que se registraron fueron muy marcados como podemos apreciarlo en el caso ilustrado, en el cual no solo hubo aumento en la intensidad, sino también en el número de contracciones durante la hora de registro. (Fig. $\mathrm{N}^{0} 5$ )
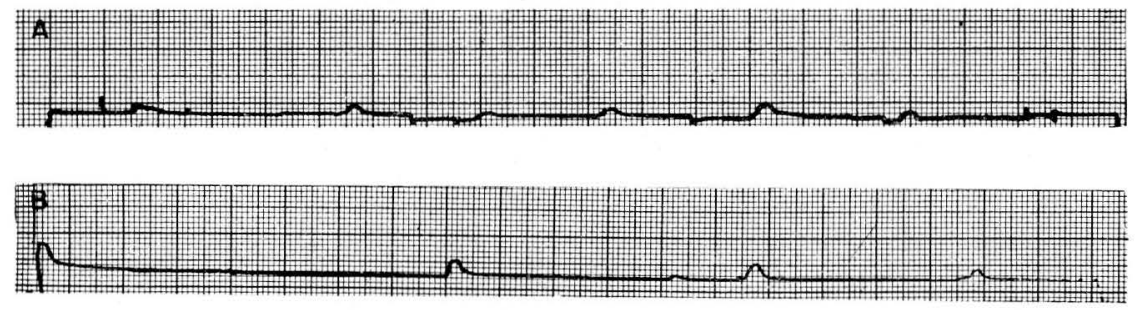

FíguRA 4 - Gráfica del paciente $N^{9} 10$. El trazado A fue hecho 8 días antes del trabajo. El trazado B fue hecho 7 días antes del trabajo y 20 horas después de la administración de $60 \mathrm{mg}$. de Propera. Nótese el aumento del tono de los parietes $(2.6 \mathrm{~mm}$.) en el trazado B después de la administración del esteroide.

En general, podemos decir, que la intensidad de las contracciones disminuyó, en la mitad de los casos analizados. Tabla ํㅜ 1.

Duración: en 7 casos las contracciones fueron cortas y no muy intensas, en 4, no hubo ninguna modificación; y en 6 de las pacientes las contracciories fueron más largas, pero de menor intensidad que las registradas durante los controles.

Intervalo: el intervalo aumentó en 7 casos, no se modificó en 8 ; y en los dos casos restartes fue más corto.

Contracciones sentidas por la paciente: en general, no se apreciaron mayores cambios subjetivos en las contracciones sentidas por la enferma, después de la adminiestración de la hormona. Solo en muy pocos casos, como mencionamos anteriormente, se presentó una sensación de tensión alrededor del abdomen.

\section{CLASIFICACION}

Para una mayor comprensión de los diferentes resultados obtenidos con esta droga, hemos clasificado arbitrariamente su efectividad, en los siguientes grupos: 
1) Efecto completo: En este grupo están incluídas las enfermas en las cuales hubo una supresión completa de las contracciones, después de administrada la droga: o que sin haber sido completa, hubo una disminución en la frecuencia y en la intensidad de la misma. Llenando estos requisitos encontramos 7 casos uno de los cuales se ilustra en la figura $N^{0} 6$.

2) Efecto incompleto: En este grupo están incluídas las enfermas que no presentaron modificaciones en el número de contracciones, pero en las cuales hubo una disminución de la intensidad, de la duración o de ambas. En este grupo quedaron comprendidos 4 casos.

3) Acción contraria: En esta, hemos agrupado los casos en los cuales hubo un aumento tanto en la frecuencia como en la intensidad de las contracciones. Con esta respuesta sólo obtuvimos un caso (Fig. $\mathrm{N}^{0} 5$ ).
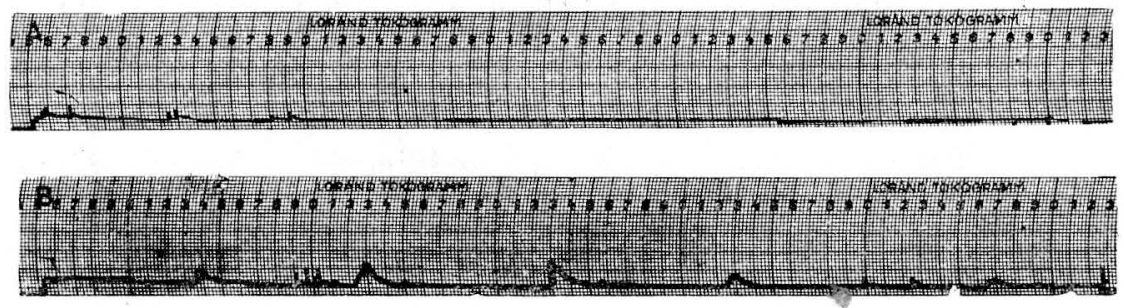

FIGURA 5 - Gráfica del paciente No 5. El trazado A fue hecho 13 días antes del parto. El trazado B fue hecho 12 días antes del parto y 24 horas después de la administración de $50 \mathrm{mg}$. de Provera. Nótese la casi completa inactividad del útero en el trazado A. Aumento marcado en la intensidad y en el número de contracciones durante la hora de registro después de la administración del esteroide en el trazado $B$.

4) Ningún efecto: En este grupo se comprende el resto de las pacientes que no pudieron ser incluídas en los grupos precedentes, y aquellas que no presentaron contracciones para ser comparadas.

Hallazgos en la excreción de los esteroides urinarios

Después de la administración oral de 6 alfa metil 17 alfa hidroxy progesterona acetato en las dosis usadas, no se registraron cambios consistentes en la excreción urinaria de los 17 ketosteroides, 17 hidroxy corticcides, pregnandiol y pregnanetriol.

Estos datos pueden observarse en la Tabla $\mathrm{N}^{0} 2$. 


\section{TABLA II}

Excreción de Esteroides Urinarios antes y después del Acetato de 6 alfa-metil 17 alfa hidroxy progesterona

\begin{tabular}{|c|c|c|c|c|c|c|c|c|c|}
\hline \multirow{3}{*}{$\begin{array}{l}\text { Paciente } \\
\mathrm{N}^{0}\end{array}$} & \multirow{3}{*}{$\begin{array}{c}\text { Dosis to- } \\
\text { tal en } \\
\mathrm{mg} \text {. }\end{array}$} & \multicolumn{8}{|c|}{ Exereción urinaria en miligramos por 24 horas } \\
\hline & & \multicolumn{2}{|c|}{ Pregnanetriol } & \multicolumn{2}{|c|}{ Pregnanediol } & \multicolumn{2}{|c|}{ 17-Ketosteroides } & \multicolumn{2}{|c|}{17 Hydroxycorticoides } \\
\hline & & Antes & Después & Antes & Después & Antes & Después & Antes & Después \\
\hline 5 & 50 & 1.3 & 0.5 & 86.2 & 16.2 & 16.7 & 13.8 & 7.9 & 4.8 \\
\hline 7 & 50 & 1.0 & 2.1 & 5.8 & 18.7 & 11.9 & 5.6 & 6.3 & 10.9 \\
\hline 8 & 60 & 1.3 & 0.5 & 9.9 & 10.3 & 11.4 & 9.8 & 4.0 & 2.9 \\
\hline 9 & 60 & 1.1 & 0.9 & 18.5 & 6.7 & 26.1 & 33.4 & 4.0 & 5.0 \\
\hline 10 & 60 & 1.2 & 1.0 & 51.2 & 30.6 & 14.3 & 16.6 & 2.5 & 4.9 \\
\hline 14 & 100 & 1.4 & 1.2 & 37.4 & 47.1 & 18.7 & 19.8 & - & - \\
\hline 15 & 100 & 0.5 & 1.1 & 32.9 & 33.6 & 15.2 & 15.9 & 3.2 & 4.8 \\
\hline
\end{tabular}


Administración del esteroide y su relación con el trabajo y el parto

De las veinticuatro pacientes estudiadas, 9 fueron primíparas y 15 multíparas. Todas ellas tuvieron trabajos de parto sin complicaciones. El trabajo de parto comenzó desde 2 hasta 32 días después de la administración del esteroide con un término me-
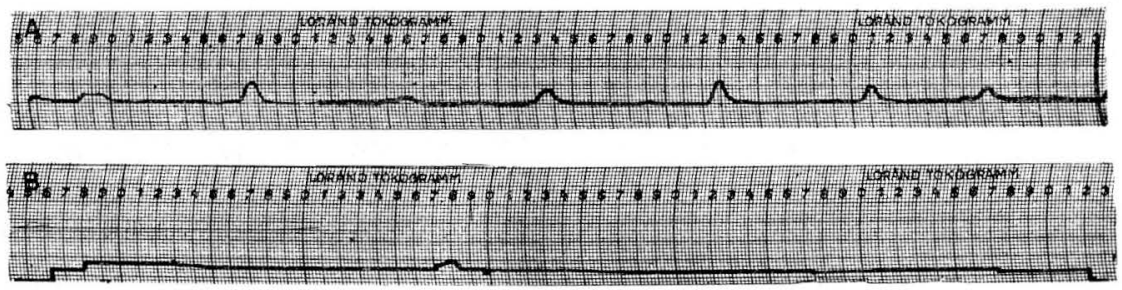

FIGURA 6 - Grafica del paciente $\mathrm{N}^{\circ}$ 6. El trazado A fue hecho 4 días antes del trabajo. El trazado $B$ fue hecho 3 días antes del trabajo y 24 horas después de la administración de $50 \mathrm{mg}$. de Provera. Nótese la muy buena actividad del útero en el trazado A mostrando contracciones regulares de Braxton-Hicks. En el trazado B después de la administración del esteroide se observa una notoria supresión de las contracciones y descenso en el tono de los parietes.

dio de 16 días. La duración del trabajo fue de 14 horas para las primigestantes y 8 horas para las multíparas. Las 24 pacientes dieron a luz 24 infantes normales de los cuales 16 fueron niñas sin signos de virilización.

El útero se contrajo bien en cada paciente, tanto durante el trabajo de parto como en el tercer período del mismo. La pérdida sanguínea durante el alumbramiento fue de un término medio de 250 c.c. de sangre. No hubo relación entre la respuesta del esteroide y la paridad de la paciente.

\section{COMENTARIO}

El presente estudio nos sugiere que la acción del acetato de 6 alfa-metil 17 alfa-hidroxy progesterona sobre el músculo uterino, no se ejerce en forma definida a pesar de las diferentes dosis administradis. Sinembargo, es evidente que en varios casos obtuvimos distintos grados de supresión, pero la diversidad de resultados nos ha hecho creer, que cada una de las pacientes responde en forma distinta. Posiblemente por las características propias del músculo uterino, su propio balance hormonal y las diferentes concentraciones de iones existentes en el tejido muscular. Seguramente, la variedad de resultados pueda ser explicada a través 
de una cuidadosa evaluación del efecto de la progesterona sobre la contracción del músculo uterino. En general, puede aceptarse que la progesterona producida por el trofoblasto en el tejido placentario, ejerce su acción localmente sobre las fibras uterinas; acción que luego se extiende por difusión al resto del órgano. Lógicamente asumimos, que el "bloqueo" ejercido por la progesterona sobre la contracción es más completo cerca de la implantación placentaria, que lejos de ella. Cuando la producción de progesterona declina, hay predominio estrogénico produciéndose, por lo tanto, una ruptura del "bloqueo progestacional". Una vez roto este "bloqueo", el útero recupera rápidamente su excitabilidad y su poder farmacológico de reacción. De lo anterior podemos deducir, que el efecto de la progesterona depende de la cantidad producida por el trofoblasto placentario, como también de la relación estrógenos-progesterona, presente en el tejido muscular.

Davis y Plotz, ${ }^{10}$ usando progesterona irradiada, demostraron que la hormona inyectada se difunde rápidamente desde el plasma sanguíneo hacia los tejidos principalmente al tejido graso, para luégo regresar lentamente a la circulación y ser excretada por el hígado y el riñón.

Parece que la progesterona actúa sobre el miometrio en una forma indirecta, o sea a través de sus dos isómeros (delta 4- $3 \mathrm{Ke}$ topregnane- 20 alfa-ol y delta- 4- 3 Ketopregnane- 20 beta-ol). Sinembargo. ninguna de estas substancias ha sido encontrada en el músculo o la mucosa del útero grávido. ${ }^{35}$

La progesterona oral, como la 6 alfa metil 17 alfa hidroxy progesterona acetato, tiene una acción retardada, requiriendo no menos de 20 horas para ejercer un efecto terapéutico de consideración. Sabemos que la potencia de la droga mencionada es por lo menos dos o más veces más efectiva que la progesterona común en su acción sobre el endometrio. ${ }^{14}$

No podemos explicar en forma acertada, los diferentes resultados obteniảos con esta hormona sobre las contracciones del útero grávido; no obstante, nos inclinamos a creer que en los casos en los cuales obtuvimos una respuesta dudosa o negativa muy posiblemente se haya debido a los altos niveles estrogénicos presentes en ciertas pacientes, los que posiblemente influyeron en estos casos. Aún pensamos que cuando se quiera lograr un bloqueo progestacional, esta nueva progesterona puede ensayarse admi- 
nistrándola en dosis no menores de 50 mlg., tratando de mantener esta dosis diariamente, durante algún tiempo y luego disminuyendo gradualmente la dosis con el objeto de evitar que una suspensión brusca de la droga traiga consigo una inversión de la relación estrógenos-progesterona y la subsecuente aparición de contracciones indeseadas.

Las determinaciones de la excreción hormonal practicadas, fallaron en demostrar un cambio notorio después de la administración de 6 alfa metil 17 alfa hidroxy progesterona acetato. Plotz y Davis, ${ }^{28}$ notaron que administrando dosis grandes de progesterona durante el embarazo, existe cierta tendencia a reducirse la excresión urinaria de androsterona y etio-colanolona; pero sin preseritarse una alteración correspondiente en la excreción del material reactivo por el Zimermman.

La excreción de estas cantidades relativamente abundantes de las substancias que registramos aquí, posiblemente representen el aumento en los 20 y 3 ketosteroides, como le es característico al embarazo avanzado (34). Bongiovani y colaboradores, ${ }^{4}$ no encontraron aumento en la excreción del pregnanetriol urinario en adultos normales, administrando 17 alfa hidroxy progesterona en cantidades comparables a las dosis empleadas en este estudio. No obstante, es muy probable que dosis mayores de 6 alfa metil 17 alfa hidroxy progesterona acetato, puedan resultar en un aumento correspondiente del pregnanetriol $\mathrm{y} / \mathrm{o}$ del pregnandiol.

\section{SUMARIO}

Dosis de 20 a $200 \mathrm{mlg}$ de Acetato de 6 alfa-metil 17 alfa-hidroxy progesterona le fueron administradas a 24 pacientes de más de 36 semanas de embarazo. Sus condiciones pre-natales fueron normales. Mediante el tocógrafo de Loránd se registró la actividad uterina y las contracciones de Braxton-Hicks antes y después de la administración del esteroide. Siete pacientes tuvieron su segundo registro antes de 20 horas y después de 36 horas de la administración de la droga. En estos casos el segundo control fué tan parecido al primero que nosotros asumimos que la efectividad del esteroide no alcanza valores óptimos antes de las 24 horas y que su efecto desaparece después de las 36 horas. Basándonos en los registros de los 17 pacientes restantes, consideramos que el efecto fue completo en 7 , incompleto en 4 , ninguno 
en 5 y un efecto contrario en el último. No hubo relación aparente entre la dosis y la respuesta obtenida; no obstante, dosis menores de $20 \mathrm{mlg}$. fueron inefectivas. En algunos pacientes el efecto depresor fue particularmente notorio sobre el número y la intensidad de las contracciones.

En la mayoría de las pacientes se manifestó un aumento en el tono (12 de 17 pacientes). La naturaleza y grado de efectividad de este nuevo esteroide son motivo todavía de mayor investigación.

Las eliminaciones urinarias de los 17 ketosteroides, 17 hidroxy corticoides, pregnandiol y pregnanetriol no se alteraron significativamente con la administración y dosis de acetato de 6 alfa-metil 17 alfa-hidroxy progesterona empleadas en este estudio.

\section{SUMMARY}

Doses of 20 to $200 \mathrm{mg}$. of 6 alpha-methyl 17 alpha-hydroxyprogesterone acetate were administered orally to 24 patients who were more than 36 weeks pregnant. All were in normal prenatal condition. Uterine activity and the Braxton-Hicks contractions were measured with the Lorand tocograph before and after administration of the steroid. The second recordings in 7 patients were made either before 20 hours or after 36 hours had elapsed. From these instances, the second recordings were so similar to the control ones that we assume that the effectiveness of the steroid does not reach optimal values before 20 hours after oral administration of this medication and disappears after 36 hours. Based on the records of the remaining 17 patients, the effect was considered complete in 7 of them and incomplete in 4 ; there was no effect in 5 , and reverse action was observed in the remaining one. There was no apparent relationship between the dosage and the response obtained; however, doses of less than $20 \mathrm{mg}$. were found to be noneffective. In some patients the depressing effect was particulary evident upon the number and strength of the contractions of late pregnancy. The tone was found to be increased in the majority of the patients, 12 out of 17 . The nature and degree of myometrial effectiveness of this new steroid, therefore, were found to be unpredictable.

Urinary levels of 17-ketosteroids, 17-hidroxycorticosteroids. pregnanediol, and of pregnanetriol were not significantly altered 
by the dosages and schedules of treatment of 6 alpha-methyl 17 alpha-hydroxyprogesterone acetate employed in this study.

RECONOCIMIENTO: Le estamos profundamente agradecidos a los Dres. Charles Hillman y William Butler, residentes de Giriecología y Obstetricia de Duke University Medical Center, 1958-1959, por su cooperación en la selección de las pacientes para este estudio. A la señora Mary R. Haddon, Secretaria y Editora. División de Endocrinología, Duke University Medical Center y a Diana de Del Corral por sus esfuerzos en la preraración de este trabajo para su publicación.

\section{REFERENCES}

1 Allen, W. M. and CORNER, G. W.: Physiology of corpus luteum. III. Normal growth and implantation of embryos after very early ablation of the ovaries, under the influence of extracts of the corpus luteum, Am. J. Physiol. 88:340-346 (March) 1929.

2 BENGTSSON L. P.: The effect of pitocin on the placenta, Am. J. Obst. \& Gynec. 74:518-520 (Sep.) 1957.

3 BONGIOV'ANNI, A. M. and EBERLEIN, W. R.: Critical analysis of methods for measurement of pregnane-3-alpha, 17-alpha, 20-alphatriol in human urine, Analytical Chem. 30:388-393, 1958.

4 BONGIOVANNI, A. M.; EBERLEIN, W. R., and CARA. J.: Studies on the metabolism of adrenal steroids in the adrenogenital syndrome, J. Clin. Endocrinol \& Metab. 14:409-422 (April) 1954.

5 BROUHA, L. and SIMMONNET, H.: Action du liquide folliculaire sur la contractilite uterine, Comp. Rend. Soc. de Biol. 96:96-97 (Jan.) 1927 .

6 CORNER, G. W.: Cyclic variation in uterine and tubal contraction waves, Am. J. Anat. 32:345-351 (Nov.) 1923.

7 CSAPO, A.: Actomyosin formation by estrogen action. Am. J. Physiol. 162·406-410. (Aug.) 1950 .

8 CSAPO, A.: Progesterone "block", Am. J. Ant. 98:273-291 (March) 1956.

9 CSAPO, A.: The relation of threshold to the $\mathbf{K}$ gradient in the myometrium, J. Physiol. 133:145-158 (July 27) 1956.

10 DAVIS, M. E. and PLOTZ, E. J.: Hormones in human reproduction. II. Further investigation of steroid metabolism in human pregnancy. Am. J. Obst. \& Gynec. 76:939-954 (Nov.) 1958.

11 ENGELHARDT, W. A. ard LJUBIMOWA, M. N.: Myosine and adenosinetri-phosphatase, Nature. 144:668-669 (Oct. 14) 1939. 
12 FALLS, F. H.; LACKNER, J. B., and KRONH, L.: Effect of progestin and estrogenic substance on human uterine contraction. J. A. M. A. 106:271-275 (Jan. 25) 1936.

13 GLENN, E. M., and NELSON, D. H.: Chemical method for the determination of 17-hidroxy-corticosteroids and 17-ketosteroid in urine following hydrolysis with beta-glucuronidase, J. Clin. Endocrinol. \& Metab. 13:911-921 (Aug.) 1953.

14 HAMBLEN, B. C. et al.: Unpublished data.

15 HEUSSER, H.; ENGEL, CH. R.; HERZIG, P. The. and PLATTNER, P1. A.: Uber steroide und sexualhormone. Uber 17-methyl-progesterone $A$, ein hoch aktives gestagen, und seine bereitung aus 21-bzw. 17-halogen-pregnenolon. Helv. Chim. Acta. 33:2229-2236, 1950.

16 HOLTORFF, A. F. and KOCH, F. C.: The colorimetric estimation of 17 -ketosteroids and their application to urine extracts. J. Biol. Chem. 135:377-392 (Sep.) 1940.

17 HORVATH, B.: Ovarian hormones and the ionic balance of uterine muscle, Proc. Nat. Acad. Science. 40:515-521, 1954.

18 KEYE, J. D.: Periodic variations in spontaneous contractions of uterine muscle, in relation to the oestrous cycle and early pregnancy, Bull. Johns Hopkins Hospital 34: 60-63 (Feb.) 1923.

19 KNAUS, H.: Zur frage der standardisation des corpus luteum-extractes. Arch. f. Exp. Path. u Pharmakol. 151: 371-380 (June-July) 1930.

20 MAKEPEASE, A. W.; CORNER, G. W., and Allen, W. M.: The effect of progestin on the in vitro response of the rabbit's uterus to pituitrin, Am. J. Physiol. 115: 376-385 (April) 1936.

21 MANZI, L.: Azione inibente degli estratti di corpo luteo sul travaglio provocato dagli estratti di lobo posteriore dell, ipofisi, nelle varie epoche della gravidanza, ricerche sperimentali. Arch. di Ostet. e Ginec. 19:220-232 (May) 1932.

22 MARDONES, E.; IGLESIAS, R., and LIPSCHUTZ, A.: Physiological action of 19-norprogesterone in the guinea pig, Poc. Soc. Exper. Biol. \& Med. 86: 451-413 (July) 1914.

23 MEYSTRE, CH.; TSCHOPP, E., and WETTSTEIN, A.: Uber steroide. Uber 11-dehydroprogesterone, das wirksamste gestagen. Helv. Chim. Acta 31:1463-1469, 1948.

24 MURPHY, D. P.: Measurements of uterine contractions in late pregnancy. A study of five patients with the Lorand tocograph. Surg. Gynec. \& Obst. 70:129-135 (Feb. 1940). 
25 MURPHY, D. P.: Uterine Contractility in Pregnancy. A Study of the Contractions of Pregnancy and Labor Under Normal and Experimental Conditions, J. B. Lippicott Co., Publisher, Philadelphia, 1947.

26 OLSEN, A. G.; SALHANICK, H. A., and HISAW, F. L.: The inhibitory action of various steroids on the Hooker-Forbes assay for progesterone, Endocrinology. 51:519-523 (Dec.) 1952.

27 PARKES, A. S. and BELlERBY, C. W.: Studies on the internal secretions of the ovary. II. The effects of injections of the oestrus producing hormone during pregnancy, J. Physiol. 62:145-155 (Dec. 10) 1926.

28 PLOTंZ, B. J. and DAVIS, M. E.: The excretion of neutral steroids in the urine of pregnant women following the administration of large doses of progesterone, Acta Endocrinol. 21:259-267 (March) 1956.

29 REYNOLDS, S. R. M. and FRIEDMAN, M. H.: Studies on the uterus. III. Activity of the uterine fistula in unanesthetized rabbits following coitus and during pseudopregnancy, Am. J. Physiol. 94:696-704 (Sev.) 1930.

30 ROBSON, J. M. and SHARAF, A. A.: Effects of some water soluble steroids on the uterus, J. Endocrinol. 8:133-137 (April) 1952.

31 STEMPFEL, R. S.: Unpublished data.

32 STRAUB F. B.: "Actin" IN Studies from the Institute of Medical Chemistry, Univ. of Szeged, Hungary, ed. by A. Szent-Gyorgy, vol. II, 1942, pp. 3-15, S. Karger, Publisher, Basel and New York.

33 SZENT-GyORGY, A.: Studies on muscle. Acta Physiol. Scandinav. vol. 9, Supplementum XXV, Stockholm, 1945.

34 VENNING, E. H.: Adrenal function in pregnancy. Endocrinology 39: 203-220 (Sep.) 1946.

35 ZANDER, J.; FORBES, T. R.; VON MUNSTERMANN, A. M., and NEHER, R.: Delta 4-3 ketopregnane-20 alpha-01 and delta 4-3-ketopregnene-20 beta ol, two naturally ocurring metabolites of progesterone. Isolation. identification, biologic activity and concentration in human tissues. J. Clin. Endocrinol. \& Metab. 18:337-353 (April) 1958.

36 ZARROW, M. X. and NEHER, G. M.: Concentration of progestin in the serum of the rabbit during pregnancy, the puerperium and following castration. Endocrinology. 56:1-8 (Jan.) 1955.

37 ZONDEK, B. and ASCHHEIM, S.: Ovulation in der graviditat-ausgelost durch hypophysenvonderlappenhormon. Endokrinologie. 1: 10-22 (Jan.) 1928. 